\title{
Photobiokatalyse
}

\section{Cyanobakterien als Biokatalysatoren}

\author{
MARC M. NOWACZYK ${ }^{1}$, HANNA C. GRIMM ${ }^{2}$, LEEN ASSIL-COMPANIONI ${ }^{2}$ UND ROBERT KOURIST $^{2}$ \\ 1 LEHRSTUHL FÜR DIE BIOCHEMIE DER PFLANZEN, RUHR-UNIVERSITÄT BOCHUM \\ 2 INSTITUT FÜR MOLEKULARE BIOTECHNOLOGIE, TU GRAZ, ÖSTERREICH
}

\section{The highly optimized natural process of oxygenic photosynthesis leads to the formation of redox equivalents, such as NADPH, that can be used to fuel heterologous biotransformations in phototrophic microorganisms. We investigated the reduction of 2-methylmaleimide by the ene-reductase YqjM in the cyanobacterium Synechocystis sp. PCC 6803 and doubled the productivity of the cells by inactivating flavodiironproteins (FDPs) as competing electron sink under self- shading conditions, reaching $18.3 \mathrm{mmol} \mathrm{h}^{-1} \mathrm{~L}^{-1}$.}

DOI: $10.1007 / \mathrm{s} 12268-021-1527-3$

(C) Die Autoren 2021

Oxidoreduktasen spielen eine wichtige Rolle in der Enzymkatalyse zur Herstellung von Feinchemikalien und Pharmastoffen. Enzymatische Redoxreaktionen benötigen externe Ko-Faktoren wie NAD(P)H, deren Preis den Produktpreis weit übersteigt. Daher erfolgt eine Regeneration meist über den Einsatz von organischen Ko-Substraten wie Isopropanol oder Glucose. Ihre stöchiometrische Verwendung und die Nutzung von nur wenigen der verfügbaren Elektronenpaare des Donormoleküls ist im Hinblick auf die Atomökonomie des Prozesses problematisch.

Cyanobakterien, Algen und Pflanzen sind photosynthetische Organismen, die Lichtenergie in chemisch gebundene Energie umwandeln können. Insbesondere die Fixierung von $\mathrm{CO}_{2}$ zum Aufbau von Biomasse im
Rahmen der Photosynthese ist dabei von Bedeutung. Erst durch die Aktivität photosynthetischer Organismen, insbesondere durch die der Cyanobakterien, hat sich Sauerstoff in der Atmosphäre angereichert, wodurch das Leben, so wie wir es heute kennen, möglich wurde.

Auf molekularer Ebene spielen Membranproteinkomplexe die entscheidende Rolle in diesem Prozess. Die lichtgetriebene Wasseroxidation wird durch das hochkomplexe Enzym Photosystem II (PSII) katalysiert. Dieser Membranproteinkomplex besteht aus mehr als 20 Untereinheiten und vielen verschiedenen Ko-Faktoren (z. B. Chlorophyll A, Pheophytin, $\beta$-Carotin, Plastochinon etc.), darunter auch der einzigartige $\mathrm{Mn}_{4} \mathrm{CaO}_{5}$ Cluster, der für eine hocheffiziente Wasserspaltung verantwortlich ist [1].
Die Elektronen aus der Wasserspaltung werden von PSII über den Plastochinonpool, den Cytochrom- $\mathrm{b}_{6}$ f-Komplex und Plastocyanin auf Photosystem I übertragen, wobei ein vektorieller Protonengradient aufgebaut wird, der z. B. zur Synthese von ATP genutzt wird. Photosystem I dient als „Elektronenpumpe“, da hier die Elektronen mithilfe von Lichtenergie auf ein höheres Energieniveau gebracht werden, sodass Ferredoxin (Fd) als zentraler Knotenpunkt zur Verteilung von Elektronen im Metabolismus reduziert werden kann, wobei hauptsächlich NADPH mithilfe der Fd-NADPH-Oxidoreduktase generiert wird.

Oxygene Photosynthese bietet ein hohes Potenzial zur Regenerierung von reduziertem Fd und NADPH. Biotransformationen in Wildtyp-Zellen von Cyanobakterien sind limitiert durch die relativ geringe Expression der Gene der nativen Oxidoreduktasen, was die Produktivität der Zellen und die RaumZeit-Ausbeute dieser Rektionen begrenzt. Die Verfügbarkeit gentechnischer Werkzeuge für Cyanobakterien ermöglicht die rekombinante Herstellung heterologer Oxidoreduktasen und damit eine erhebliche Steigerung der Produktivität [2]. Im Jahr 2016 konnten wir zeigen, dass Zellen von Synechocystis sp. PCC 6803 (Synechocystis) mit dem Gen der Enreduktase YqjM von B. subtilis die C-C-
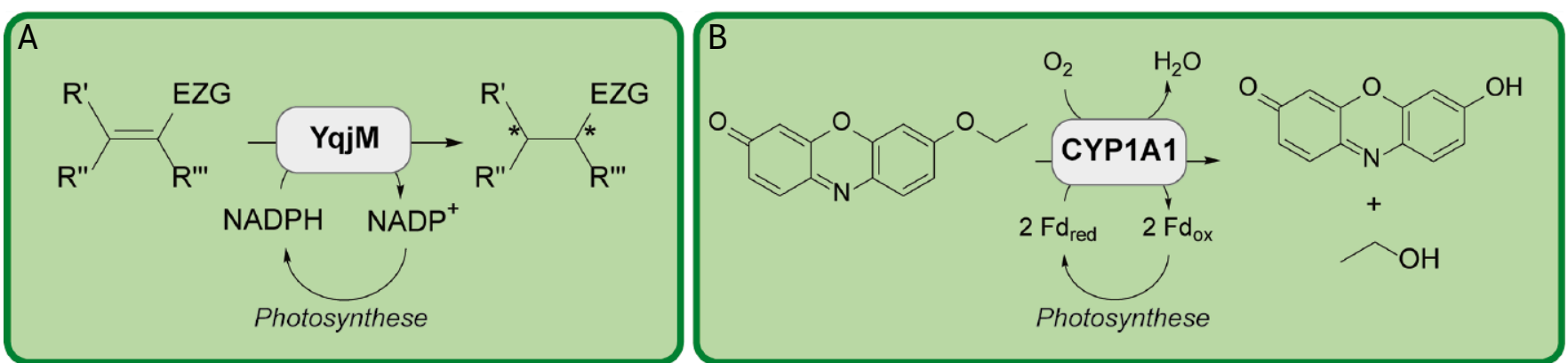

A Abb. 1: Beispiele für Ganz-Zell-Biotransformationen in rekombinanten Cyanobakterien. A, Die En-Reduktase YqjM von Bacillus subtilis katalysiert die Reduktion von C=C-Doppelbindungen in Synechocystis sp. PCC 6803 [3, 9]. Der benötigte Ko-Faktor kann über Photosynthese regeneriert werden. B, Das Gen der P450-Monooxygenase CYP1A1 aus Rattus norvegicus wurde in das Genom von Synechococcus sp. PCC 7002 integriert [11]. Die Bildung des fluoreszierenden Produkts Resofurin kann photometrisch verfolgt werden. Ferredoxin (Fd) dient als Elektronenshuttle und bezieht diese aus der photosynthetischen Maschinerie. EZG: Elektronen-ziehende Gruppe. 


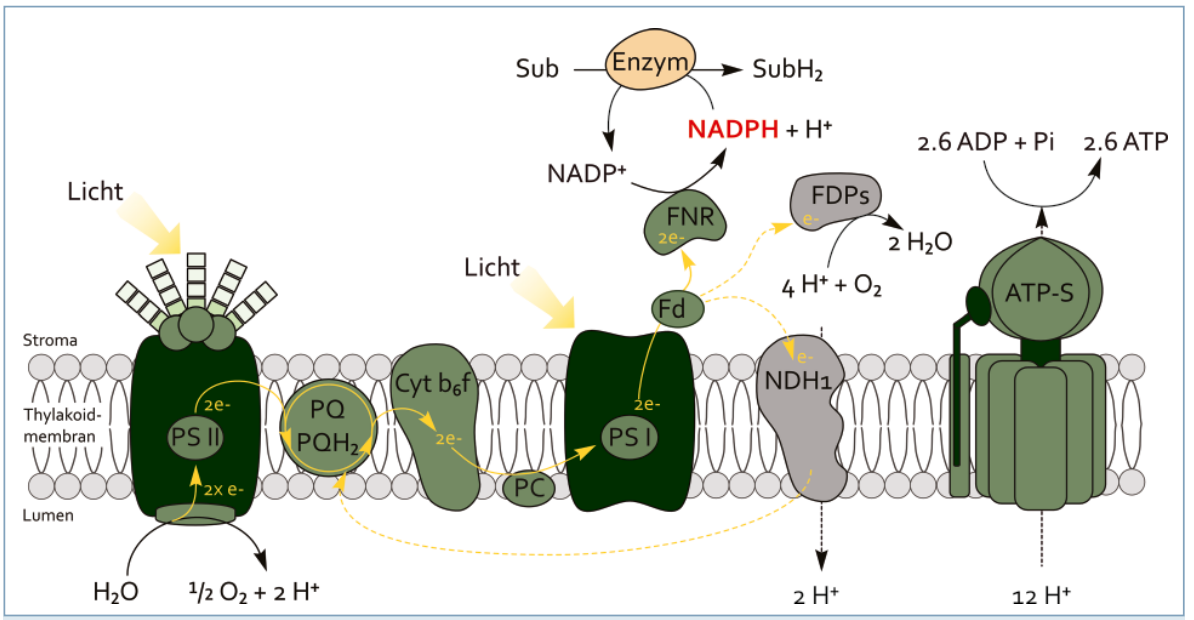

$\triangle$ Abb. 2: Vereinfachte Darstellung der Thylakoidmembran und der darin verankerten Proteinkomplexe, die in die Photosynthese involviert sind. PSII: Photosystem II; PQ: Plastochinon; $\mathrm{PQH}_{2}$ :

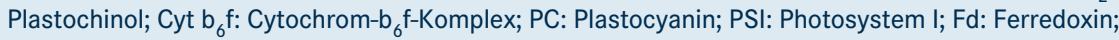
FNR: Ferredoxin-NADP+-Reduktase; FDP: Flavodiironproteine; NDH1: NADH-Dehydrogenase-Komplex 1; ATP-S: ATP-Synthase.

Doppelbindungsreduktion einer Reihe von prochiralen Substraten mit sehr vielversprechenden Produktbildungsraten von über 100 U per Gramm Zelltrockengewicht katalysieren (Abb. 1, [3]). Die lichtgetriebene Ganzzell-Biotransformation wurde seitdem auf eine ganze Reihe verschiedener Oxidoreduktasen angewandt, darunter eine Cyclohexanon-Monooxygenase aus Acinetobacter cacloaceticus NCIMB 9871 (CHMO) mit Aktivitäten von bis zu 5,7 U g ${ }_{\mathrm{DCW}}{ }^{-1}$ [4], die Monooxygenase AlkBGT aus Pseudomonas putida GP mit 5,7 U g ${ }_{\text {DCW }}{ }^{-1}$ [5], die CYP450-Monooxygenase aus Acidovorax sp. CHX100 mit $39 \mathrm{U} \mathrm{g}_{\mathrm{DCW}}{ }^{-1}[6]$ sowie eine Reihe an Iminreduktasen zur Herstellung optisch reiner Imine mit Aktivitäten bis $20 \mathrm{U} \mathrm{g}_{\mathrm{DCW}}{ }^{-1}$ [7]. Diese Beispiele unterstreichen die allgemeine Anwendbarkeit des Ansatzes und die Tatsache, dass spezifische Aktivitäten in der Größenordnung etablierter Produktionssysteme erreicht werden können.

Eine sehr große Herausforderung bei der Etablierung photobiokatalytischer Prozesse ist die Beleuchtung der Zellen in Photobioreaktoren [8], da die photosynthetische Aktivität von Cyanobakterien von der Lichtverfügbarkeit abhängt. Dies betrifft sowohl die Kultivierung der rekombinanten Cyanobakterien als auch ihre Anwendung, denn die starke Absorption der Zellen limitiert die Zelldichten für Photobiotransformationen auf wenige Gramm per Liter [3, 9]. Eine zu hohe Intensität der Lichteinstrahlung führt aber zu Photoinhibition und zu starken Lichtfluktuationen im Photobioreaktor. Eine Steigerung der volumetrischen Produktion durch eine Erhöhung der Zelldichte, ein Routineverfahren bei heterotrophen Bakterien und Hefen, ist daher nur sehr bedingt möglich. Eine erfolgreiche Anwendung der Ko-FaktorRegenerierung durch katalytische Wasserspaltung der Photosynthese erfordert daher eine erhebliche Steigerung der spezifischen Produktbildungsraten von Cyanobakterien.

Dies veranlasste uns, die limitierenden Faktoren - wie die intrazelluläre Enzymkonzentration einer cyanobakteriellen Photobiotransformation - am Beispiel der Enreduktase YqjM, bislang die Reaktion mit der höchsten Reaktionsrate, genauer zu untersuchen [9]. Bei einem Vergleich der zwei Promotoren $\mathrm{P}_{p s b A 2}$ und $\mathrm{P}_{c p c}$ wurde mit letzterem eine 1,7fach höhere intrazelluläre Enzym- konzentration und eine 1,3fach höhere Aktivität des Ganzzell-Biokatalysators erzielt (Abb. 3, [9]). Während dies die Bedeutung der Enzymkonzentration unterstreicht, legt es auch nahe, dass andere Faktoren bei hohen Reaktionsraten limitierend werden.

Der Mechanismus von YqjM läuft über zwei Schritte ab. Im ersten, oxidativen Schritt, reduziert ein Molekül NADPH den internen Ko-Faktor Flavinmononukleotid (FMN). Nach Ablösung des NADPHs bindet ein Substratmolekül, was die Reduktion der $\mathrm{C}=\mathrm{C}$-Doppelbindung im reduktiven Schritt einleitet [9, 10]. Eine Analyse der kinetischen Parameter der Enreduktase in der NADPH-abhängigen Reduktion von 2-Methylmaleimid zu (R)-2-Methylsuccinimid zeigte eine mindestens 50fach höhere turnoverKonstante für den reduktiven Schritt [9]. Der $\mathrm{K}_{\mathrm{D}}$-Wert von rund $40 \mu \mathrm{M}$ des oxidativen Reaktionsschritts gegenüber NADPH führt bei intrazellulären Konzentrationen unter $500 \mu \mathrm{M}$ zu einer geringeren Enzymaktivität. Der $\mathrm{k}_{\text {cat }}$-Wert für NADPH ist darüber hinaus 12 fach höher als der für NADH, sodass NADPH als metabolische Elektronenquelle angenommen werden kann [9]. Diese Ergebnisse legten den Schluss nahe, dass für diese Reaktion die Verfügbarkeit des photosynthetischen NADPH limitierend ist.

Eine mögliche Senkung der intrazellulären NADPH-Konzentration wurde über PAMNADPH-Fluoreszenz untersucht: Spektroskopische Techniken, insbesondere optische Spektroskopie, spielen schon seit Beginn der Photosyntheseforschung eine große Rolle zur Charakterisierung von Elektronentransportprozessen in der photosynthetischen Zelle,

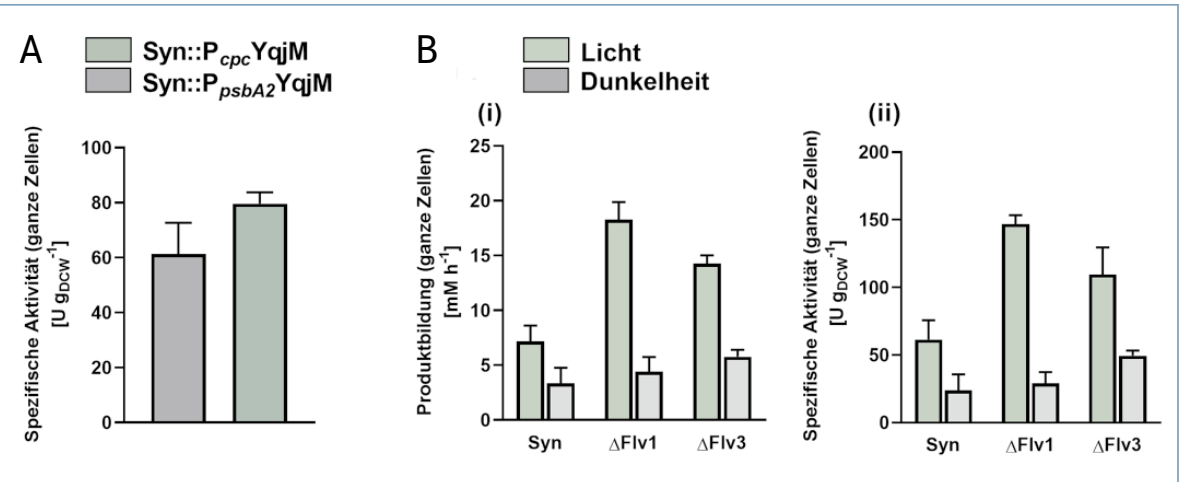

A Abb. 3: Ganz-Zell-Biotransformationen mit Synechocystis-Zellen. Das Enzym YajM wird rekombinant in den Zellen produziert und katalysiert die Spaltung von 2-Methylmaleimid zu 2-Methylsuccinimid. A, Einfluss des Promotors $\left(P_{p s b A 2}\right.$ oder $\left.P_{c p c}\right)$ vor dem YajM-Gen auf die Aktivität der Zellen. Durch den stärkeren $P_{\text {cpc }}$-Promotor wird mehr YqjM gebildet, was die Aktivität der Zellen steigert. B, Aktivität und Produktbildung, wenn die Expressionskassette für YqjM in die Knock-outStämme $\Delta$ Flv1 und $\triangle F$ Flv3 eingebracht wurde. Diese FDPs stellen eine Elektronensenke dar. Ihr gezieltes Ausschalten verbessert den Elektronenfluss zur heterologen Reaktion [9]. 
da z. B. über die zeitaufgelöste Messung der PSII-Chlorophyllfluoreszenz der Redoxzustand des PQ-Pools verfolgt werden kann. In der Tat zeigte es sich, dass die Reduktion des Substrats während der Biotransformation eine Senkung der intrazellulären NADPHKonzentration zur Folge hatte. Die Ergebnisse führten zu dem Schluss, dass die photosynthetische NADPH-Produktion nicht ausreicht, um den erhöhten NADPH-Konsum durch Biotransformationen bei hohen Reaktionsraten zu kompensieren. Eine Erhöhung der NADPH-Zufuhr durch das Ausschalten konkurrierender Konsumenten gewinnt dadurch an Bedeutung.

Der produzierte Pool an reduzierten Reaktionsäquivalenten kann den Bedarf der Zelle für metabolische Prozesse übersteigen und die Entstehung von reaktiven Sauerstoffspezies und Photoinhibition könnten die Folge sein [11]. Aus diesem Grund wird die Zelle durch verschiedene Mechanismen geschützt, die als „Elektronensenken“ bezeichnet werden. So katalysiert der NAD(P)H-Dehydrogenase-ähnliche Komplex 1 (NDH 1) die Oxidation von Fd und den Rücktransport der Elektronen zum Plastochinon (PO) (Abb. 2, [11, 12]). Dieser zyklische Elektronentransport kann verhindert werden, indem die Funktionalität der D2-Untereinheit des NDH-1-Komplexes gestört wird [11]. Stattdessen können die Elektronen auf eine heterologe Reaktion umgelenkt werden, wie das Beispiel der P450-Monooxygenase CYP1A1 in Synechococcus sp. PCC 7002 zeigt (Abb. 1). Der Knock-out-Stamm $\triangle$ ndhD2 wies dabei eine erhöhte Aktivität und höhere Photosyntheserate auf als der Vergleichsstamm mit intaktem NDH-1-Komplex [11].

Eine weitere Elektronensenke sind Flavodiironproteine (FDPs), die überschüssige Elektronen von reduziertem Fd [13] auf Sauerstoff übertragen (Abb. 2). Da das Photosystem II durch Wasserspaltung Elektronen und Sauerstoff generiert und bei der FDP-katalysierten Reaktion Wasser entsteht, spricht man von einem Wasser-Wasser-Zyklus [14]. Unter bestimmten Bedingungen werden so bis zu 60 Prozent der photosynthetisch entstandenen Elektronen zurückgeleitet [15]. FDPs sind für das Überleben der Cyanobakterien unter natürlichen Lebensbedingungen mit fluktuierendem Licht essenziell, können unter kontrollierten Kultivierungsbedingungen jedoch inaktiviert werden [16]. Um die Elektronen, die über den Wasser-WasserZyklus verloren gehen, auf die heterologe Reaktion umzuleiten, brachten wir das Gen für YqjM in die beiden FDP-Knock-out-Stämme $\Delta$ Flv1 und $\Delta$ Flv3 ein. Diese können kein funktionales Flv1/Flv3-Heterooligomer bilden. Biotransformationen mit beiden Knockout-Stämmen zeigten eine deutliche Steigerung der Produktivität der Zellen und der Produktbildung. Eine Produktbildungsrate von $18 \mathrm{mM} / \mathrm{h}$ unterstreicht, dass Cyanobakterien Redox-Biotransformationen mit hoher Aktivität katalysieren können (Abb. 3, [9]).

Diese Beispiele der lichtgetriebenen Hydroxylierung und der Enreduktase zeigen, dass die Reaktionsrate von Redox-Biotransformationen in Cyanobakterien über ein Engineering des photosynthetischen Elektronentransports erhöht werden kann. Dies gelang sowohl über eine erhöhte Bildung von NADPH bzw. $\mathrm{Fd}_{\text {red }}$ durch die Inaktivierung des NDH-1-Komplexes [11] als auch durch die Ausschaltung der Flavodiironproteine als alternative Elektronensenke [9]. In beiden Fällen konnte die NADPH-Verfügbarkeit für die Redoxreaktion als heterologe Elektronensenke deutlich erhöht werden - ein wichtiger Schritt auf dem Weg zu einer breiten Anwendung von Photobiokatalyse ohne organische Ko-Substrate in Zellen, die selbst aus Kohlendioxid und Wasser gebildet werden.

\section{Literatur}

[1] Umena Y, Kawakami K, Shen JR, Kamiya N (2011) Crystal structure of oxygen-evolving photosystem II at a resolution of $1.9 \AA$. Nature 473: 55-60

[2] Ferreira EA, Pacheco CC, Pinto F et al. (2018) Expanding the toolbox for Synechocystis sp. PCC 6803: validation of replicative vectors and characterization of a novel set of promoters. Synth Biol 3: ysy014

[3] Köninger K, Gómez Baraibar Á, Mügge C et al. (2016) Recombinant cyanobacteria for the asymmetric reduction of $\mathrm{C}=\mathrm{C}$ bonds fueled by the biocatalytic oxidation of water Angew Chemie Int Ed 55:5582-5585

[4] Böhmer S, Köninger K, Gómez Baraibar Á et al. (2017) Enzymatic oxyfunctionalization driven by photosynthetic water-splitting in the cyanobacterium Synechocystis sp. PCC 6803. Catalysts 7: 240

[5] Hoschek A, Bühler B, Schmid A (2019) Stabilization and scale-up of photosynthesis - driven $\omega$-hydroxylation of nonanoic acid methyl ester by two-liquid phase whole-cell biocatalysis. Biotechnol Bioeng 116.8:1887-1900

[6] Hoschek A, Toepel J, Hochkeppel A et al. (2019) Lightdependent and aeration-independent gram-scale hydroxylation of cyclohexane to cyclohexanol by CYP450 harboring Synechocystis sp. PCC 6803. Biotechnol J 14: 1800724 [7] Büchsenschütz HC, Vidimce-Risteski V, Eggbauer B et al. (2020) Stereoselective biotransformations of cyclic imines in recombinant cells of Synechocystis sp. PCC 6803 . ChemCatChem 12: 726-730

[8] Huang Q, Jiang F, Wang L, Yang C (2017) Design of photobioreactors for mass cultivation of photosynthetic organisms. Engineering 3: 318-329

[9] Assil-Companioni L, Büchsenschütz HC, Solymosi D et al. (2020) Engineering of NADPH supply boosts photosynthesisdriven biotransformations. ACS Catal 10: 11864-11877 [10] Pesic M, Fernández-Fueyo E, Hollmann F (2017) Characterization of the old yellow enzyme homolog from Bacillus subtilis (YqjM). ChemistrySelect 2: 3866-3871 [11] Berepiki A, Gittins JR, Moore CM, Bibby TS (2018) Rational engineering of photosynthetic electron flux enhances light-powered cytochrome P450 activity. Synth Biol 3: ysy009 [12] Schuller JM, Birrell JA, Tanaka H et al. (2019) Structural adaptations of photosynthetic complex I enable ferredoxin-dependent electron transfer. Science 363:257-260 [13] Sétif P, Shimakawa G, Krieger-Liszkay A, Miyake C (2020) Identification of the electron donor to flavodiiron proteins in Synechocystis sp. PCC 6803 by in vivo spectroscopy. Biochim Biophys Acta Bioenerg 1861: 148256

[14] Helman Y, Tchernov D, Reinhold L et al. (2003) Genes encoding A-type flavoproteins are essential for photoreduction of $\mathrm{O} 2$ in cyanobacteria. Curr Biol 13: 230-235

[15] Allahverdiyeva Y, Ermakova M, Eisenhut M et al. (2011) Interplay between flavodiiron proteins and photorespiration in Synechocystis sp. PCC 6803. J Biol Chem 286: 24007-24014 [16] Jokel M, Nagy V, Tóth SZ et al. (2019) Elimination of the flavodiiron electron sink facilitates long-term H2 photoproduction in green algae. Biotechnol Biofuels 12: 1-16

Funding note: Open Access funding provided by Graz University of Technology. Open Access: Dieser Artikel wird unter der Creative Commons Namensnenn Mearbeitung, Verbreitung und Wiedergabe in jeglichem Medium und Formg Bearbeitung, Verbreitung und Wiedergabe in jeglichem Medium und For
erlaubt, sofern Sie den/die ursprünglichen Autor(en) und die Quelle ordnungsgemäß nennen, einen Link zur Creative Commons Lizenz beifügen und ordnungsgemä nennen, einen Link zur Creative Commons Lizenz beifu
angeben, ob Änderungen vorgenommen wurden. Die in diesem Artikel angeben, ob Anderungen vorgenommen wurden. Die in diesem Artikel
enthaltenen Bilder und sonstiges Drittmaterial unterliegen ebenfalls der genannten Creative Commons Lizenz, sofern sich aus der Abbildungslegende nichts anderes ergibt. Sofern das betreffende Material nicht unter der genannten Creative Commons Lizenz steht und die betreffende Handlung nich nach gesetzlichen Vorschriften erlaubt ist, ist für die oben aufgeführten Weiterverwendungen des Materials die Einwilligung des jeweiligen Rechteinhabers einzuholen. Weitere Details zur Lizenz entnehmen Sie bitte der
Lizenzinformation auf http://creativecommons.org/licenses/by/4.0/deed.de.
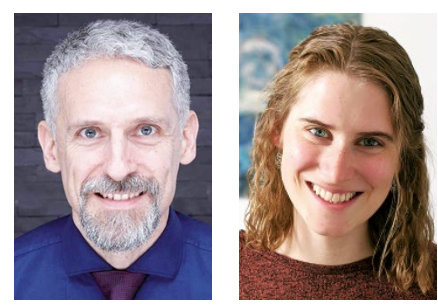

Marc M. Nowaczyk und Hanna C. Grimm

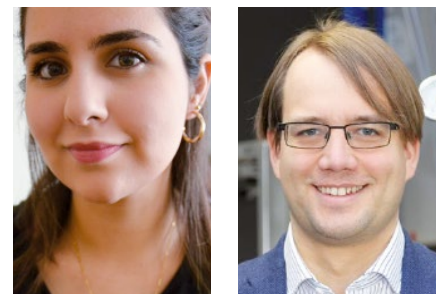

Leen Assil-Companioni und Robert Kourist

Korrespondenzadresse:

Prof. Dr. Robert Kourist

Institut für Molekulare Biotechnologie

TU Graz

Petersgasse 14

A-8010 Graz

kourist@tugraz.at

www.tugraz.at/institute/imbt/home 